\title{
IL-22 defines a novel immune pathway of antifungal resistance
}

\author{
A De Luca ${ }^{1,3}$, T Zelante ${ }^{1,3}$, C D’Angelo $^{1}$, S Zagarella ${ }^{1}$, F Fallarino ${ }^{1}$, A Spreca $^{1}$, RG Iannitti ${ }^{1}$, P Bonifazi ${ }^{1}$, \\ $\mathrm{J}-C ~ R e n a u l d^{2}$, F Bistoni ${ }^{1}$, P Puccetti $^{1}$ and L Romani ${ }^{1}$
}

The role of IL-17 and Th17 cells in immunity vs. pathology associated with the human commensal Candida albicans remains controversial. Both positive and negative effects on immune resistance have been attributed to IL-17/Th17 in experimental candidiasis. In this study, we provide evidence that IL-22, which is also produced by Th17 cells, has a critical, first-line defense in candidiasis by controlling the growth of infecting yeasts as well as by contributing to the host's epithelial integrity in the absence of acquired Th1-type immunity. The two pathways are reciprocally regulated, and IL-22 is upregulated under Th1 deficiency conditions and vice versa. Whereas both IL-17A and F are dispensable for antifungal resistance, IL-22 mediates protection in IL-17RA-deficient mice, in which IL-17A contributes to disease susceptibility. Thus, our findings suggest that protective immunity to candidiasis is made up of a staged response involving an early, IL-22-dominated response followed by Th1/Treg reactivity that will prevent fungal dissemination and supply memory.

\section{INTRODUCTION}

Candida albicans is a human commensal of mucosal and skin surfaces. A number of dysregulated immune reactivities has been associated with the fungus and/or certain fungal components. ${ }^{1}$ We have shown that the activation of Th17 cells at sites of infection may contribute to inflammatory pathology and worsens fungal diseases. ${ }^{2}$ However, the role of IL-17 and Th17 cells in immunity vs. pathology in candidiasis remains controversial. ${ }^{3}$ Although Th17 are present in the human T cell memory repertoire to the fungus ${ }^{4}$ and defective Th17 cell differentiation has been linked to mucocutaneous candidiasis in patients with primary immunodeficiencies, ${ }^{5-7}$ the role of IL-17/Th17 in human candidiasis is not so unambiguously defined. Similarly, both positive and negative effects on immune resistance have been attributed to Th17 and IL-17 receptor (IL-17R) signaling in experimental candidiasis. ${ }^{2,8-10}$

Given the importance of IL-17 in recruiting myeloid cells to sites of infection and because defective neutrophil recruitment underlined the susceptibility of IL-17R-deficient mice to disseminated ${ }^{9} \mathrm{or} \mathrm{oral}^{8}$ candidiasis, it is assumed that the very absence of Th17 cells is directly responsible for the increased susceptibility to candidiasis. Somehow paradoxical, however, administration of exogenous IL-17 not only did not rescue the Th17 deficiency in oral candidiasis but induced systemic inflammatory effects. ${ }^{8}$ We have found that neutralization of IL-17A greatly reduced fungal burden, and corrected inflammation while concomitantly reducing neutrophil recruitment and Th17 cell activation. ${ }^{2,11}$ The mechanisms that linked innate immunity and inflammation to chronic infection have been credited to the offending potential of IL-17A that, although promoting neutrophil recruitment, impeded the timely restriction of neutrophil inflammatory potential, thus preventing optimal protection to occur. ${ }^{12}$

Th17 cells also produce IL-22, a member of the IL-10 family of cytokines, which has been shown to have a more important role than IL-17 in host defense in the lung and gut. ${ }^{13-15}$ However, the role of IL-22 in inflammatory responses has been confounded by data suggesting both pro- and anti-inflammatory functions. The finding that IL-23 was protective in condition of IFN- $\gamma$ deficiency ${ }^{2}$ suggest that IL-23 may regulate the production of cytokines other than IL-17A/IL-17F. Herein, we provide evidence that IL-22 has a first-line defense in candidiasis by critically controlling initial fungal growth and epithelial homeostasis in the relative absence of Th1 immunity. The two pathways are reciprocally regulated, being the IL-23/IL-22 pathway upregulated in Th1-deficiency and the Th1 pathway in IL-22 deficiency, and compensate each other in the relative absence of either one.

\footnotetext{
${ }^{1}$ Department of Experimental Medicine and Biochemical Sciences, University of Perugia, Perugia, Italy. ${ }^{2}$ Ludwig Institute for Cancer Research Ltd Experimental Medicine Unit, Universite Catholique de Louvain, Brussels, Belgium. ${ }^{3}$ These authors contributed equally to this work. Correspondence: L Romani (Iromani@unipg.it) 
Although both IL-17A and F are dispensable in infection, IL-22 mediates antifungal resistance in IL-17RA-deficient mice, in which IL-17A contributes to disease susceptibility. Thus, our findings suggest that protective immunity to candidiasis is made up of a staged response involving an early, IL-22-dominated response followed by $\mathrm{Th} 1 /$ Treg reactivity that will prevent fungal dissemination and supply memory.

\section{RESULTS}

\section{IL-22 is produced in candidiasis and directly targets epithelial cells}

IL-23 is produced by dendritic cells (DCs) in response to C. albicans yeasts ${ }^{16}$ and hyphae ${ }^{4}$ and activates inflammatory Th17 cells. ${ }^{2}$ We looked whether IL-23 induces the production of IL-22 in mice intragastrically infected with C. albicans and its dependency on fungal morphotype. IL-22, protein and transcript, IL-23 protein and IL-22R transcript were induced in the stomach, particularly after yeast infection (Figure 1a). All these cytokines were rapidly induced, with peak expression around day 4 of infection, and were also detected in colon, liver and kidneys (Supplementary Figure S1a). DCs and CD3-NKp46+ cells are known to produce IL-22 in the gut. ${ }^{15,17-21}$ We found that IL-22 was produced by DCs (Figure 1b) and NKp46 + cells (Figure 1c) from Peyer's patches but not by F4/80 + or Gr-1 + cells (data not shown). Purified NKp46 + cells from Peyer's patches produced IL-22 upon acute stimulation with C. albicans and IL-23 in vitro (Figure 1d). Further analysis revealed that NKp46 + cells but not NKp46- cells constitutively and in infection expressed retinoic acid-related orphan receptor (ROR) $\gamma \mathrm{t}$, IL-23R, aryl hydrocarbon receptor, crucially required for IL-22 production by Th17 cells, ${ }^{22}$ CCR5, CCR7, IL-22 but not IL-17A transcripts (Supplementary Figure S1b), indicating their similarity to gut ROR $\gamma \mathrm{t}+\mathrm{NKp} 46+$ cells $^{15,17-19}$ and cryptopatch lymphoid tissue inducer-like cells ${ }^{23}$ that are expanded by commensals and zymosan and crucially control epithelial integrity. Infection also expanded IL-22 + cells (from 3-5\% of naïve to $8-10 \%$ vs. $35-40 \%$, yeasts vs. hyphae) in mesenteric lymph nodes 7 days after infection, as revealed by intracellular cytokine staining (Figure 1e). In yeast infection, IL-22 + cells were mostly CD3- (Figure 1e), much expanded at two (from $4-5 \%$ to $20-25 \%$, naive vs. infected mice) than 7 (Figure 1e) days after the infection and poorly co-expressed IL-17A (4-6\%) (Figure 1e). In contrast, in addition to CD3-, IL-22 + CD4 + cells were expanded in infection with hyphae and co-expressed IL-17A (40-45\%). These data indicate that IL-22 is produced by innate and adaptive immune cells in candidiasis.

IL-22, together with IL-17, induces a number of antimicrobial peptides at mucosal sites, such as the antibacterial Reg proteins ${ }^{24}$ and anticandidal S100 proteins, S100A8 and S100A9. ${ }^{25}$ STAT3 (signal transducer and activator of transcription 3 ) is a key regulator of antimicrobial peptides induced by IL-22. ${ }^{26} \mathrm{To}$ assess whether IL-22 directly targets gut epithelial cells to induce antimicrobial responses via STAT3, we looked first whether C. albicans associates with gut epithelial cells in infection and then whether phosphorylation of STAT3 and production of antimicrobial peptides occur. Electron microscopy revealed that
C. albicans yeasts and hyphae both penetrate gut epithelial cells early in infection, with yeasts eventually residing within gut crypts later on in infection (Figure 1f). Concomitantly, phosphorylation of STAT3 occurred in epithelial cells in vivo (Figure 1g) and in vitro on purified epithelial cells exposed to C. albicans and IL-22 (Figure $\mathbf{1 h}$ ). We also found that S100A8 and S100A9, RegIII $\gamma$, more than RegIII $\beta$, were all upregulated in infection in the stomach (Figure 1i) and colon (data not shown). RegIII $\gamma$ and RegIII $\beta$ were also upregulated in IL-17RA KO but not IL-22 KO mice, whereas S100A8 and S100A9 expression were dependent on both IL-22 and IL-17. These data suggest that IL-22 signals epithelial cells for STAT3 activation, induction of Reg proteins and, together with IL-17, S100A proteins.

\section{IL-23 regulates IL-22 production in candidiasis}

To define the role of innate IL-22 production, we infected recombination-activating gene 2 deficient (Rag2 $\mathrm{KO}$ ) that owing to their lack of $\mathrm{T}$ and $\mathrm{B}$ cells must rely on innate defense mechanisms of antifungal resistance. Rag2 $\mathrm{KO}$ mice were more resistant than WT mice to mucosal candidiasis in the early phase, but became very susceptible to infection at a later time point, as judged by the high fungal burden in the stomach and dissemination to the esophagus (Figure 2a). IL-22 was greatly produced in Rag2 $\mathrm{KO}$ mice (Figure $\mathbf{2 b}$ ) and accounted for innate resistance to infection, as its neutralization increased fungal growth (Figure 2a). Depletion of NK1.1 + cells also resulted in increased fungal growth (data not shown), confirming that NKp46 + CD3 - cells, present in Rag2 KO mice, ${ }^{17,18}$ contribute to innate IL-22 production in candidiasis.

To assess whether IL-23 regulates innate IL- 22 production, we measured IL-22 and IL-17A production in IL-23 p19 KO or IL-12 p35 KO mice infected with C. albicans. We found that the production of IL-22 and IL-17A was lower in p19 KO mice than WT mice (Figure 2c) and was associated with a reduced ability to limit the fungal growth early in infection in these mice (Figure 2e). In contrast, the production of IL-22 and IL-17A was higher in p35 KO than WT mice (Figure 2d) and was associated with increased control of fungal growth early in infection (Figure 2e). Blocking IL-23 and IL-22 decreased resistance, particularly to yeasts, but blocking IL-17A increased resistance to hyphae (Figure 2f), a finding indicating that IL-23 acts through IL-22. The finding that, late in infection, resistance was increased in p19 KO mice and decreased in p35 $\mathrm{KO}$ (Figure 2e), also indicates that mechanisms of antifungal resistance are different in early and late infection, likely contingent upon the fungal burden. These data suggest a dual role for IL-23 early in infection. Beside expanding Th17 effector cells, ${ }^{2}$ IL-23 also regulates IL-22 production, which likely has a protective role in the early phase of the infection.

\section{IL-22 serves a protective role in candidiasis}

To directly assess the role of IL-22 in candidiasis, we inoculated IL-22 KO mice ${ }^{27}$ with C. albicans yeasts or hyphae intravenously or with C. albicans hyphae intragastrically. Although mice survived infections (data not shown), an increased fungal burden 

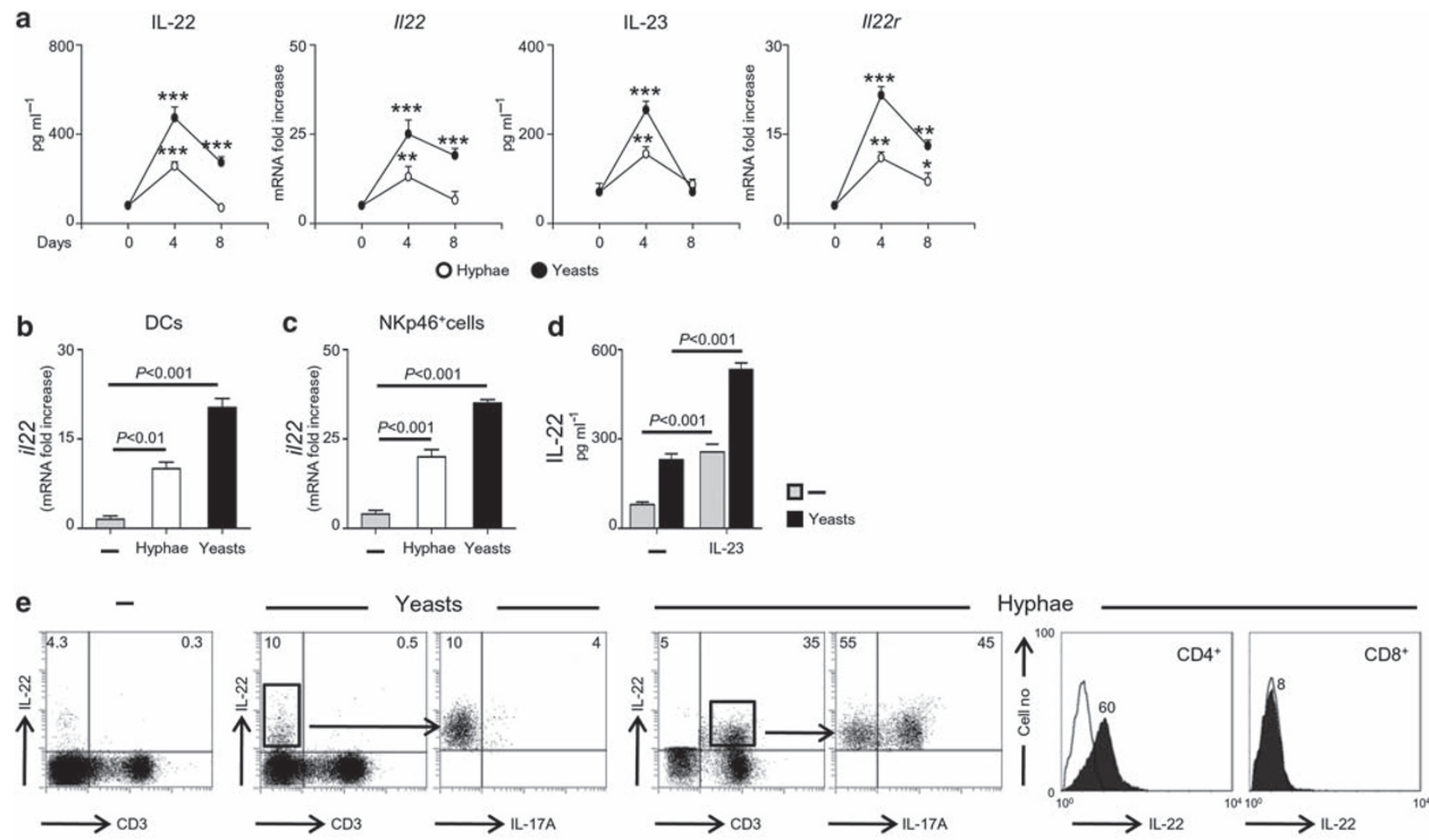

\section{f}
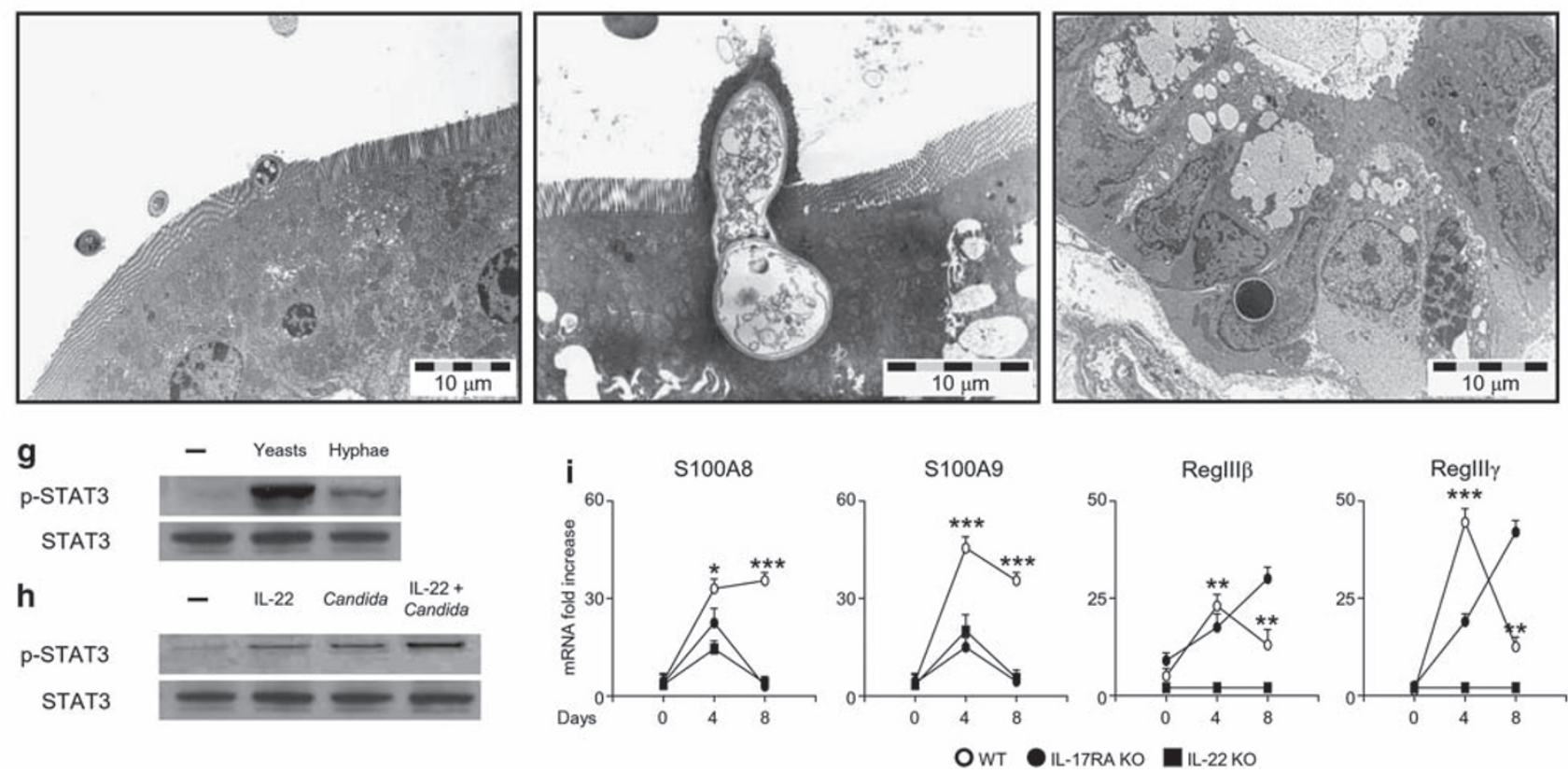

Figure 1 IL-22 production in murine candidiasis. (a) Time-course real-time RT-PCR analysis of $i$ i22/il22r in the stomach and ELISA assays for IL-22 and IL-23 in stomach homogenates of C57BL/6 mice after the intragastric infection with $C$. albicans yeasts or hyphae. ${ }^{*} P<0.05$, ${ }^{* *} P<0.01$, ${ }^{\star \star \star} P<0.001$, infected vs. uninfected (0) mice. Real-time RT-PCR analysis of il22 in Peyer's patches (b) CD11c+ CD103+DCs or (c) CD3-NKp46+ cells a day after the infection. (d) IL-22 production by naïve CD3-NKp46 + exposed to C. albicans yeasts in vitro, with and without (-) $20 \mathrm{ng}$ rIL-23. (e) Intracellular staining of IL-22 in CD3- stained mesenteric lymph node a week after infection with yeasts or hyphae. Intracellular IL-17A staining was done on gated IL-22+ positive cells. Numbers indicate percent cells in gate. Histograms showing IL-22+ cells in purified CD4+ or CD $8+$ cells (far right panels). (f) Transmission electron microscopy of gut of mice with $C$. albicans infection. After $4 \mathrm{~h}$ of inoculation, note the interaction of yeasts with villi (left, magnification, $\times 2,800$ ), the penetration of hyphae through the epithelial barrier (middle, magnification $\times 4,600$ ) and $8 \mathrm{~h}$ later, yeasts inside crypts (right, magnification $\times 3,600$ ). bar $=10 \mu \mathrm{m}$. (g) Western blotting showing that STAT3 phosphorylation occurred in colonic epithelial cells a day after infection with yeasts or hyphae and (h) in epithelial cells exposed to C. albicans yeasts in vitro, in the presence of $20 \mathrm{ng}$ rlL-22 for $15 \mathrm{~min}$. p-STAT3, phosphorylated (activated) STAT3. (i) Time-course real-time RT-PCR analysis of antimicrobial S100A peptides and Reg proteins in the stomach of mice infected with $C$. albicans. ${ }^{*} P<0.05,{ }^{* *} P<0.01,{ }^{* *} P<0.001$, C57BL/6 vs. IL-17RA KO and IL-22 KO mice (S100A) vs. IL-22 KO (Reg proteins). All data are representative of two to four experiments. 

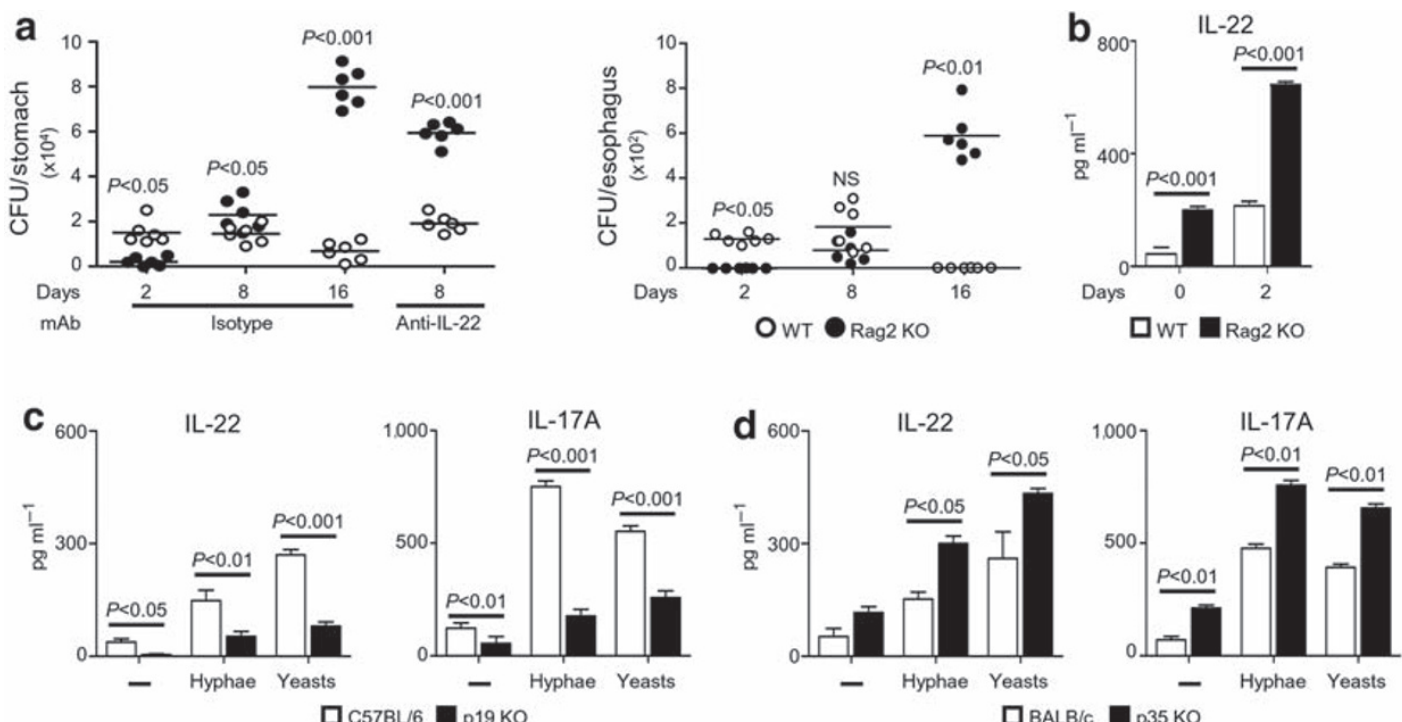

$\mathbf{\square}$ c578L6 $\mathbf{Q}_{\mathrm{p} 19 \text { Ko }}$
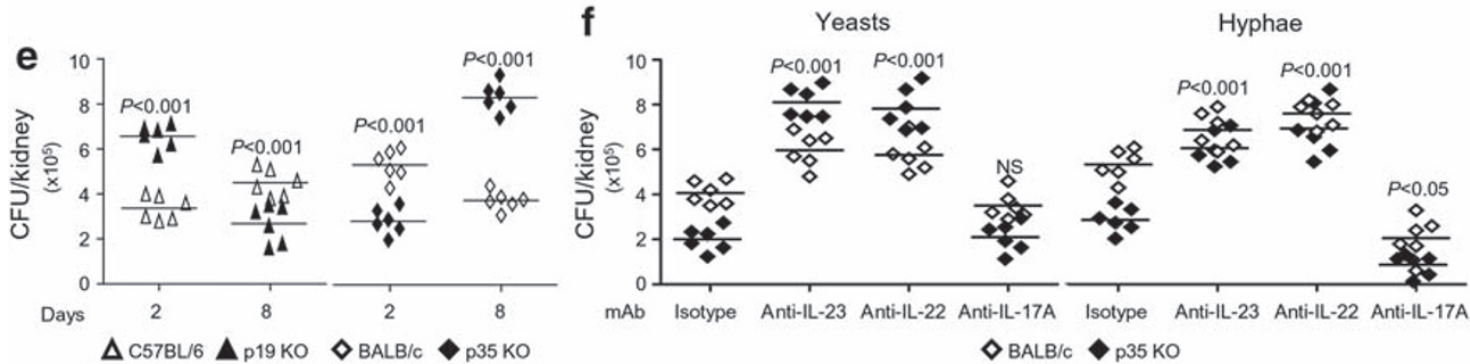

Figure 2 IL-23 regulates IL-22 production in candidiasis. (a) Colony-forming units (CFU \pm S.e.) in the stomach and esophagus of C57BL/6 (WT; $n=6$ ) and Rag2 KO $(n=6)$ mice infected intragastrically with $C$. albicans hyphae. Mice received $300 \mu \mathrm{g}$ intraperitoneally of IL-22-neutralizing or isotype $\mathrm{mAb}$ on days 0 and 1 after the infection. $P, \mathrm{CFU}$ in KO vs. WT mice and anti-IL-22-treated vs. isotype-treated mice. Data are representative of two experiments. (b) Levels of IL-22 (ELISA) in the stomach homogenates of WT and KO mice at 2 days after the infection. Levels of IL-22 and IL-17A in the kidney homogenates of IL-23 p19 KO (c) and IL-12 p35 KO (d) mice 3 days after the intravenous infection with $C$. albicans yeasts or hyphae.

(e) Fungal growth in the kidneys of C57BL/6 or BALB/c WT $(n=6)$, IL-23 p19 KO $(n=6)$ and IL-12 p35 KO $(n=6)$ mice infected intravenously with $5 \times 10^{5}$ C. albicans hyphae. $P$, CFU in KO vs. WT mice. (f) Fungal growth in the kidneys of p35 KO mice intraperitoneally injected with $100 \mu \mathrm{g}$ of IL-23-, IL-22- or IL-17A-neutralizing mAb the day of and a day after the intravenous infection with $C$. albicans yeasts or hyphae. $P$, mAb-treated WT or KO mice vs. isotype-treated WT or KO mice, respectively. Data are representative of two to four experiments. NS, not significant.

was observed in the kidneys in the intravenous yeast infection (Figure 3a) or in the stomach in the mucosal infection (Figure 3b) in IL-22 KO as compared with WT mice. Despite the higher fungal growth, no dissemination to the liver and kidneys (Figure 3f) was observed. Histological analysis revealed no obvious alterations of the kidney parenchyma after the intravenous infection, except for an increased number of infiltrates of mononuclear and polymorphonuclear cells and fungi in IL-22 KO mice (Figure 3a). In the mucosal model, IL-22 KO mice showed signs of mucosal hyperplasia in the stomach (Figure $3 \mathbf{b}$ ) and colon (Supplementary Figure S2a). The infection was predominantly superficial in the stomach of WT mice with limited submucosal inflammation and inflammatory cell recruitment. In contrast, hyphae penetrating the mucosal barrier (Figure 3b) were visible in IL-22 $\mathrm{KO}$ mice and were associated with a robust mononuclear inflammatory cell recruitment, a finding also confirmed by the elevated local expression of $\mathrm{Cxcl} 2$ and decreased expression of neutrophil-recruiting Cxcl1 (Figure 3b). These data indicate that IL-22 is dispensable for the control of fungal dissemination and may serve as a protective molecule that regulates inflammatory cell recruitment and barrier integrity.
The absence of fungal dissemination in the relative absence of IL-22 together with the finding that, similar to WT mice, infected IL-22 KO mice resisted subsequent re-infection (data not shown), indicates that a robust Th1 response occurs in the absence of IL-22. This was confirmed by the analysis of IL-12 and IL-23 production and Th cell activation. IL-12 production in the stomach, IFN- $\gamma$ production, Tbet and IL-12 $\beta 2 \mathrm{R}$-expressing $\mathrm{CD} 4+\mathrm{T}$ cells in mesenteric lymph nodes were all higher in IL-22 KO as compared with WT mice, whereas IL-23 and IL-17A production and Rorc- and IL-23R-expressing Th cells were lower (Figure 3c). IL-10 production and Foxp3-expressing T cells were also higher in KO than WT mice (Figure 3d). In both types of mice, Th1 cells were present a week after infection and were further expanded in infection, as already shown. ${ }^{16}$ Neutralization of IFN- $\gamma$ reduced IFN- $\gamma+$ Th1 cells, increased IL-17A + Th17 cells (Figure 3e) and favored fungal dissemination to the liver and kidneys in both types of mice (Figure 3f). These data show that Th1 cells are upregulated in IL-22-deficiency and limit fungal dissemination. All together, these results indicate that IL-22 accounts for innate resistance to candidiasis at the early stages of the infection. Data obtained with the infecting morphotype, 
a

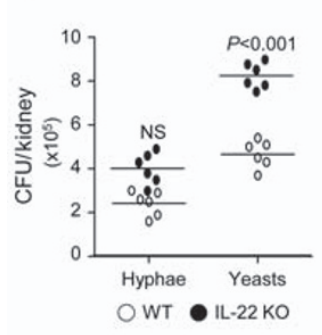

b

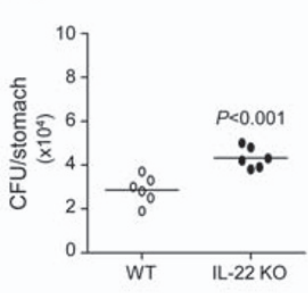

c
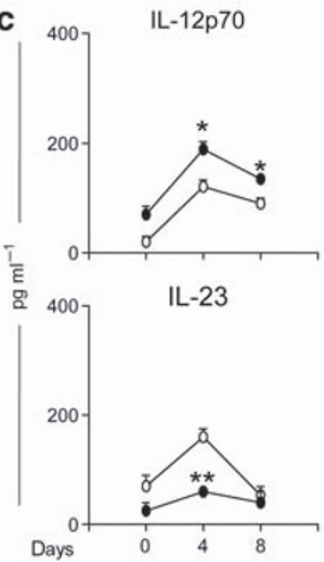

WT

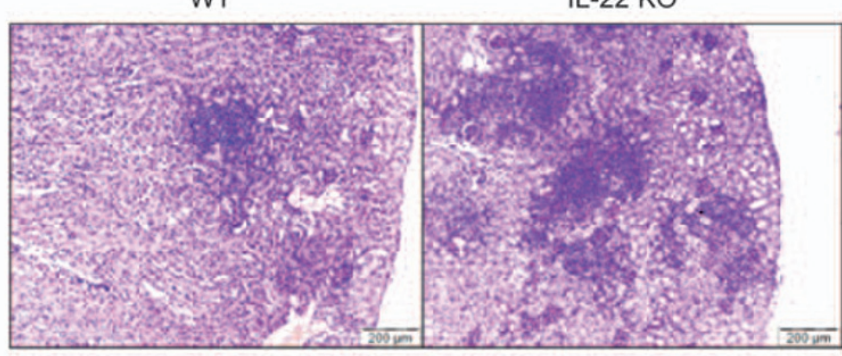

WT
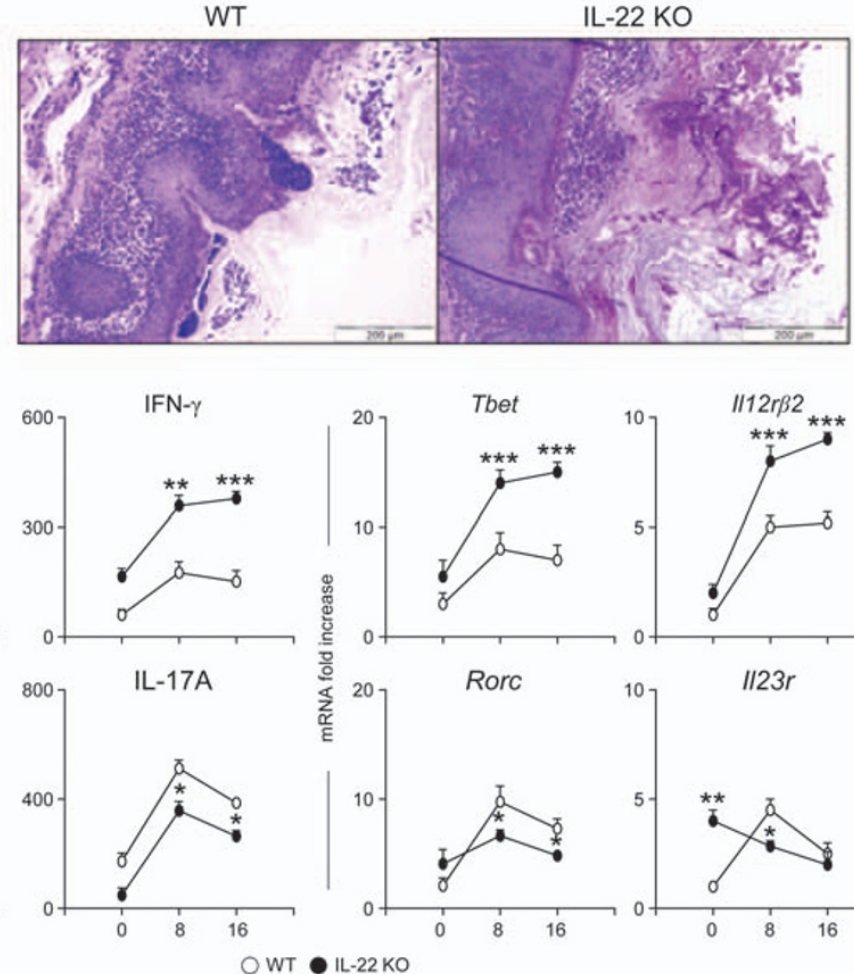
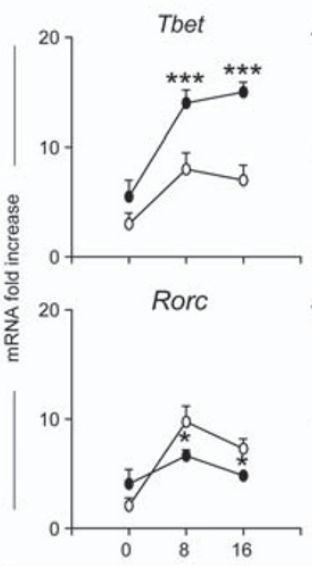
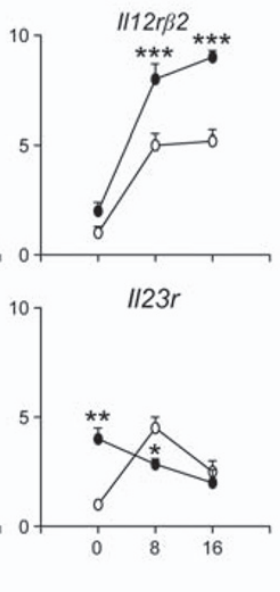

Cxc/2
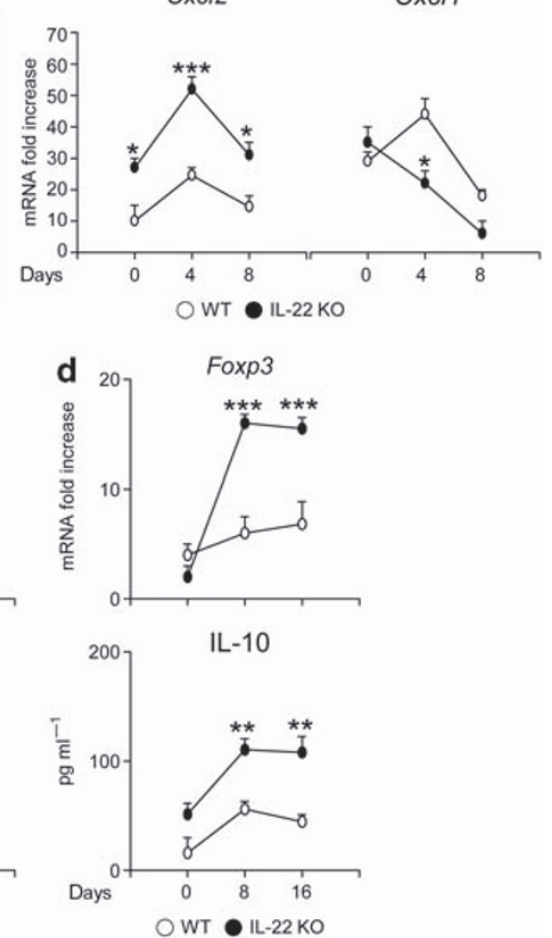

e

IFN- $\gamma$
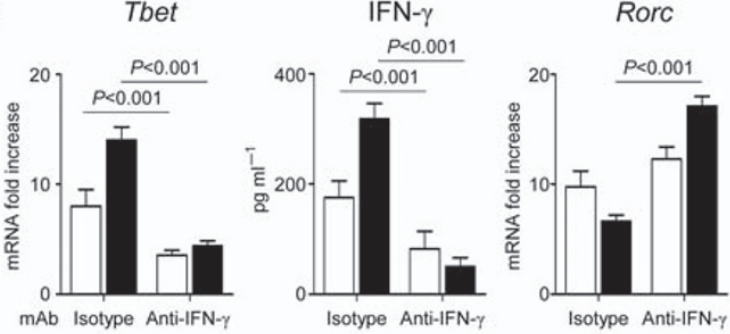

$\square$ WT IL-22 KO

IL-17A
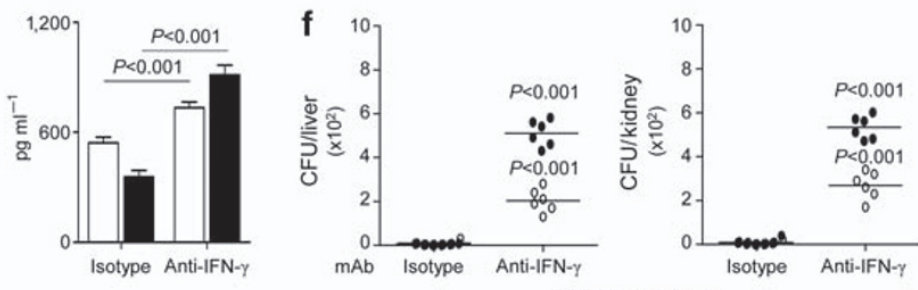

O WT - IL-22 KO

Figure 3 Crucial role of IL-22 in candidiasis. (a) Fungal growth (CFU \pm s.e.) and histology in the kidneys of C57BL/6 WT ( $n=6)$ and IL-22 KO ( $n=6)$ mice inoculated intravenously with $C$. albicans hyphae or yeasts. PAS-stained kidney sections ( 3 days after hyphae infection) showing numerous abscesses in IL-22 KO mice. (b) Fungal growth (CFU \pm s.e.), histology and expression of Cxcl2 and Cxcl1 mRNA in the stomach of C57BL/6 WT $(n=6)$ and IL-22 KO $(n=6)$ mice inoculated intragastrically with $C$. albicans hyphae. PAS-stained stomach sections (3 days after infection) showing fungal elements penetrating the mucosal barrier and inflammatory cell recruitment in IL-22 KO mice. Representative images are shown. $P$, KO vs. WT mice. (c and d) Time-course cytokine production (ELISA) and real-time RT-PCR analysis in CD4 + T cells isolated from mesenteric lymph nodes of intragastrically infected IL-22 KO and WT mice. CD4+ were restimulated with plate bound anti-CD3 $\varepsilon$ and anti-CD28 and $50 \mathrm{units} \mathrm{ml}^{-1} \mathrm{IL}-2 \mathrm{for} 48 \mathrm{~h}$ before cytokines quantification in culture supernatants. ${ }^{*} P<0.05$, ${ }^{* *} P<0.01$, ${ }^{* *} P<0.001$, KO vs. WT mice. (e) Cytokine production (ELISA), realtime RT-PCR analysis of CD4 + T cells from mesenteric lymph nodes and (f) fungal dissemination (on day 7) in IL-22 KO or WT mice treated with IFN- $\gamma$ neutralizing or isotype $\mathrm{mAb}$ the day of and 1 day after the infection. $P, \mathrm{mAb}$-treated vs. isotype-treated mice. Data are representative of four experiments.

the yeasts, confirmed the important contribution of IL-22 in early resistance to yeasts (Supplementary Figure S2b-d). In addition, the finding that resistance was less compromised in
IL-22 KO mice on the BALB/c background (Supplementary Figure S2b), suggests the importance that genetic variation of IL-22 may have in infections. 

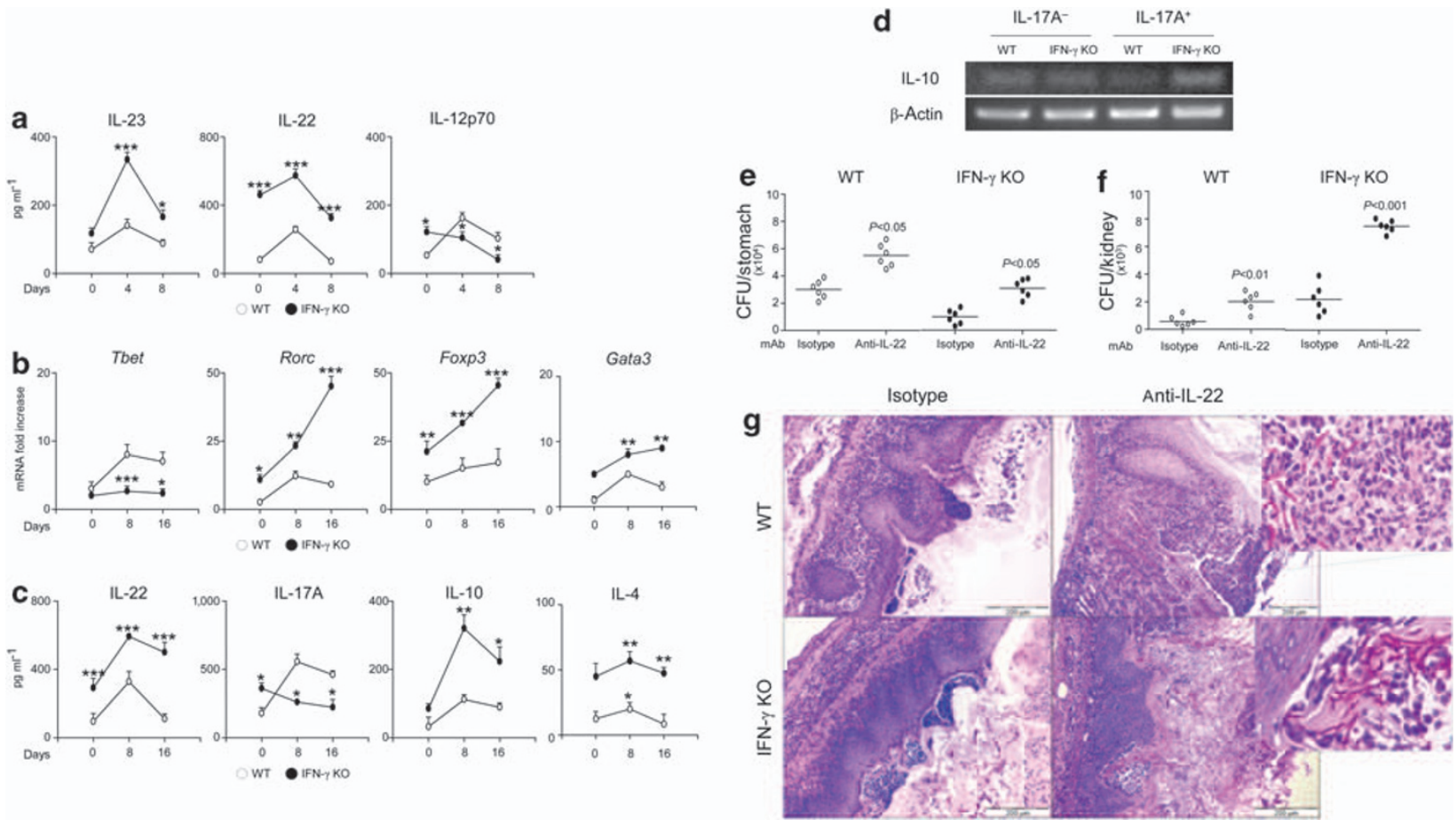

Figure 4 IL-22 provides protection in condition of Th1 deficiency. (a) Time-course cytokine production (ELISA) in the stomach, (b) real-time RT-PCR analysis and (c) cytokine production in CD3 + cells from mesenteric lymph nodes of C57BL/6 WT $(n=6)$ and IFN- $\gamma$ KO $(n=6)$ mice intragastrically inoculated with $C$. albicans hyphae. CD3+ were re-stimulated with plate bound anti-CD3 $\varepsilon$ and anti-CD28 and 50 units $\mathrm{ml}^{-1} \mathrm{IL}-2$ for $48 \mathrm{~h}$ before cytokines quantification in culture supernatants. ${ }^{\star} P<0.05,{ }^{\star \star} P<0.01,{ }^{\star \star \star} P<0.001$, KO vs. WT mice. All data are representative of four experiments. (d) RT-PCR and real-time RT-PCR analysis of IL-17- and IL-17+ cells from the mesenteric lymph nodes of WT or KO mice, after in vitro re-stimulation with PMA + ionomycin, as per the manufacturer's instruction. (e) Fungal growth (CFU \pm s.e.) in the stomach, (f) dissemination to the kidney and (g) histological analysis of PAS-stained stomach section (8 days after infection) in WT and IFN- $\gamma$ KO mice treated with IL-22 neutralizing or isotype $\mathrm{mAb}$ the day of and 1 day after the infection. IL-22 neutralization exacerbated inflammatory cell recruitment, tissue invasion (magnified in the inset) and fungal growth particularly in IFN- $\gamma \mathrm{KO}$ mice. Representative images are shown. $P$, mAb-treated vs. isotype-treated mice. $P<0.01$, IFN- $\gamma \mathrm{KO}$ vs. WT untreated mice (stomach) and $P<0.001$, IFN- $\gamma$ KO vs. WT untreated mice (kidney).

\section{IL-22 provides protection in condition of Th1 deficiency}

The upregulated Th1 response in IL-22 KO mice suggests that IL-22 and Th1 responses are reciprocally regulated in infection. To directly prove this, we measured IL-23 and IL-22 production in IFN- $\gamma$ KO mice intragastrically infected with Candida hyphae and found that peak levels of IL-23 and IL-22 were higher in KO than WT mice, whereas peak levels of IL-12 were lower (Figure 4a) and correlated with a 10-fold expansion of IL-22 + IL-23R + NKp46 + cells in the Peyer's patches (data not shown). Lower levels of Tbet but higher levels of Rorc and Foxp3 expression were observed in mesenteric lymph nodes $\mathrm{CD} 3+\mathrm{T}$ cells from IFN- $\gamma$ KO than WT mice (Figure $4 \mathbf{b}$ ) and were associated with an increased production of IL-22 and IL10 and low production of IL-17A (Figure 4c). Gata3 expression and IL-4 production were also higher in $\mathrm{KO}$ than WT mice (Figure $4 \mathbf{b}$ and c). Because IL-17 + cells also co-expressed IL10 (Figure 4d), these data suggest that Th17 cells with regulatory features ${ }^{28}$ are expanded in IFN- $\gamma$ KO mice and, similar to cells co-expressing Rorc and Foxp3 in the lamina propria, ${ }^{29}$ produce IL-22 and IL-10 more than IL-17A. Consistent with the high level of IL-22, fungal growth in the stomach was significantly lower in KO than WT mice (Figure 4e), was associated with limited dissemination to the kidneys (Figure 4f) and with superficial inflammatory cell influx and limited tissue invasion (Figure 4g). The upregulated IL-22 production was causally linked to resistance, as IL-22 neutralization increased the local fungal growth (Figure 4e), resulted in fungal dissemination (Figure 4f) and greatly exacerbated inflammatory cell recruitment and tissue invasion by fungi (Figure $\mathbf{4 g}$ ). Thus, a loss of barrier function occurs when IL-22 is neutralized. IL-22 neutralization slightly decreased the levels of IL-17A or IL-17F production (data not shown), as already described. ${ }^{30}$ However, neutralization of IL-17A and IL-17F did not modify susceptibility to infection (data not shown). These data indicate that IL-22, together with IL-23, ${ }^{2}$ has a crucial protective role in mucosal antifungal defense in condition of Th1 deficiency and that both the IL-22 and Th1 pathways are cross-regulated in infection. Despite the high resistance to mucosal infection, IFN- $\gamma \mathrm{KO}$ were less resistant than WT mice to re-infection (data not shown), a finding suggesting that IL-22 alone does not provide an efficient memory response to the fungus.

To corroborate the notion that IL-22 may serve a first-line defense against the infecting yeast morphotype, we assessed the role of IL-22 in host defense against $C$. albicans yeasts, other species of yeasts, such as C. krusei and Saccharomyces cerevisiae. We intragastrically infected WT and IFN- $\gamma \mathrm{KO}$ mice and treated them with IL-22-neutralizing antibody. The fungal burden in the stomach was lower in $\mathrm{KO}$ than WT mice and peripheral 
dissemination did not occur. However, the local fungal growth increased (Supplementary Figure S3a) and dissemination occurred, particularly in KO mice after IL-22 neutralization (Supplementary Figure S3b). These data further indicate that IL-22 may serve a first-line defense against yeast infection.

\section{IL-22 is upregulated and Th1 responses are downregulated in IL-17R-deficient mice}

Studies in IL-17RA-deficient mice have shown these mice to be susceptible to disseminated ${ }^{9}$ or oral ${ }^{8}$ candidiasis. We measured IL-22 and IL-23 production, STAT3 phosphorylation as well as the expansion of IL-22 + cells in IL-17RA KO mice infected intragastrically with C. albicans yeasts. We found that IL-23 and IL-22 productions in the stomach (Figure 5a), IL-23 and IL-22 transcripts in Peyer's patches DCs (Figure 5b) and STAT3 phosphorylation in epithelial cells (Figure 5c) were all higher in IL-17RA KO than WT mice. IL-22 + cells were also more expanded in IL-17RA KO than WT mice (Figure 5d). Transcripts of Il23r, Rorc, Il22, Il17a and Foxp3 were higher in $\mathrm{CD} 3+$ cells from KO than WT mice, whereas transcripts of $I l 12 \beta 2 r$, Tbet and If $n \gamma$ were lower (Figure 5e). On correlating these findings with susceptibility to infection, we found that IL-22-mediated resistance to yeast infection as indicated by the increased fungal growth upon blockade of IL-22 (Figure 5f) and no evidence of inflammatory pathology and dissemination in yeast infection (Figure 5i). Similar to infection with yeasts, IL-17RA KO mice were more resistant than WT mice to a low inoculum of $C$. albicans hyphae (data not shown). However, similar to what is observed in oral candidiasis, ${ }^{8}$ IL-17RA KO were more susceptible to a higher inoculum of hyphae, although they eventually recovered from the infection, albeit slower than WT mice, as judged by fungal clearance (Figure 5g), and no dissemination to the kidneys (Figure 5h). Histopathology revealed that numerous fungal hyphae and pseudohyphae, that were much longer in KO than WT mice $(41.3 \pm 5.2 \mu \mathrm{m}$ vs. $21.7 \pm 4.5 \mu \mathrm{m})$, were present in the abundant keratinized layer of the stomach in IL-17RA KO mice (Figure 5i). These data suggest that IL-22 may contribute to early resistance to infection in condition of defective IL-17R signaling, likely contingent upon the fungal morphotype (yeasts more than hyphae) and/or fungal load. In contrast, the reduced activation of Th1 responses observed in IL-17RA deficiency, ${ }^{8}$ may offer an explanation for the increased susceptibility to infection and is consistent with the importance of IL-17 in the induction of optimal antimicrobial Th1 responses, ${ }^{31}$ including to C. albicans,${ }^{10}$ and of Th17 developmental plasticity. ${ }^{32}$

\section{IL-17A and $F$ are dispensable in candidiasis}

Although local application of IL-17A failed to rescue the Th17 deficiency, ${ }^{8}$ and IL-17A KO mice are not more susceptible to systemic candidiasis, ${ }^{10}$ the ability of IL-17 to mobilize neutrophils is thought to be the principal reason why this cytokine is important for protection against pathogens and C. albicans. ${ }^{8,9}$ To directly evaluate the role of IL-17A or IL-17F in infections, we assessed IL-17A KO or IL-17F KO mice for susceptibility to mucosal candidiasis. We found that IL-17A KO mice were as resistant as WT mice to infection both in terms of fungal growth (Figure 6a) and tissue inflammatory pathology (Figure 6b). In contrast, IL-17F KO mice were more susceptible to it (Figure 6a and b) and, similar to IL-17RA KO mice, to dissemination to the esophagus. These data suggest that IL-17A, however important, is dispensable, rather than essential, ${ }^{8}$ in candidiasis and its deficiency is largely compensated for, as recently reported. ${ }^{10}$ Actually, the increased expression of IL-17A in either IL-17RA $\mathrm{KO}$ and IL-17F KO (Figure 6c) mice suggests an association of IL-17A with susceptibility to the infection. Consistent with the possible offending potential of the IL-17 family in fungal infections, ${ }^{12}$ we found that IL-17A blockade (Figure 6d) increased resistance to infection (Figure 6e) and ameliorated inflammatory pathology in either WT or IL-17RA KO mice (Figure 6f). Importantly, IL-17A neutralization also increased resistance and ameliorated pathology in IL-17F KO (Figure 6e and f), a finding indicating that IL-17F may inhibit IL-17A. Similar results were obtained in the disseminated infection. The blockade of IL-17A and IL-17F was associated with a reduced fungal growth and the influx of inflammatory cells in the kidney parenchyma (Supplementary Figure S4a). Opposite results were obtained upon IL-22 blockade (Supplementary Figure S4b). These findings confirm the protective effect of IL-22 in infection to which IL-17A may adversely contribute when present. IL-17F contributes to anticandidal resistance by inhibiting IL-17A production, as already shown, ${ }^{33}$ although additional effector functions cannot be ruled out.

\section{DISCUSSION}

This study shows that IL-22 has a more crucial role than IL-17 in mucosal host defense to C. albicans. IL-22 expression in vivo is regulated by IL-23 and accounts for innate resistance to candidiasis at the early stages of the infection in the kidney and gut. Although IL-23 has very diverse pro-inflammatory functions, ${ }^{34}$ our data clearly define that induction of IL-22 by IL- 23 is crucial for the early control of $C$. albicans infection. Thus, by expanding Th17 effector cells ${ }^{2}$ and IL-22 production by innate cells (this study), IL-23 has a dual role in infection. Although adaptive CD $4+\mathrm{T}$ cells also produce IL-22 in candidiasis, IL-22 production by DCs and NKp46 + cells account for resistance to infection in the absence of the adaptive compartment. IL-22 deficiency impairs resistance to the early infecting morphotype, the yeasts, and compromises the epithelial integrity. The massive inflammatory infiltration and pattern of chemokine expression observed in IL-22 KO mice upon infection suggest that regulation of chemokine gradients, in addition to granulopoiesis are potential effector mechanisms downstream IL-22. IL-22 directly targets gut epithelial cells to induce STAT3 phosphorylation, the Reg family proteins and, together with IL-17, S100A8 and S100A9, known to have potent anticandidal activity and antiinflammatory effects. ${ }^{25}$ We have recently shown that ablation of STAT3 decreases antifungal resistance and promotes inflammation in mucosal candidiasis. ${ }^{35}$

Our data support a model in which the IL-23/IL-22 axis controls the initial fungal growth and tissue homeostasis likely exploiting primitive anti-fungal effector defense mechanisms, ${ }^{36}$ 


\section{ARTICLES}
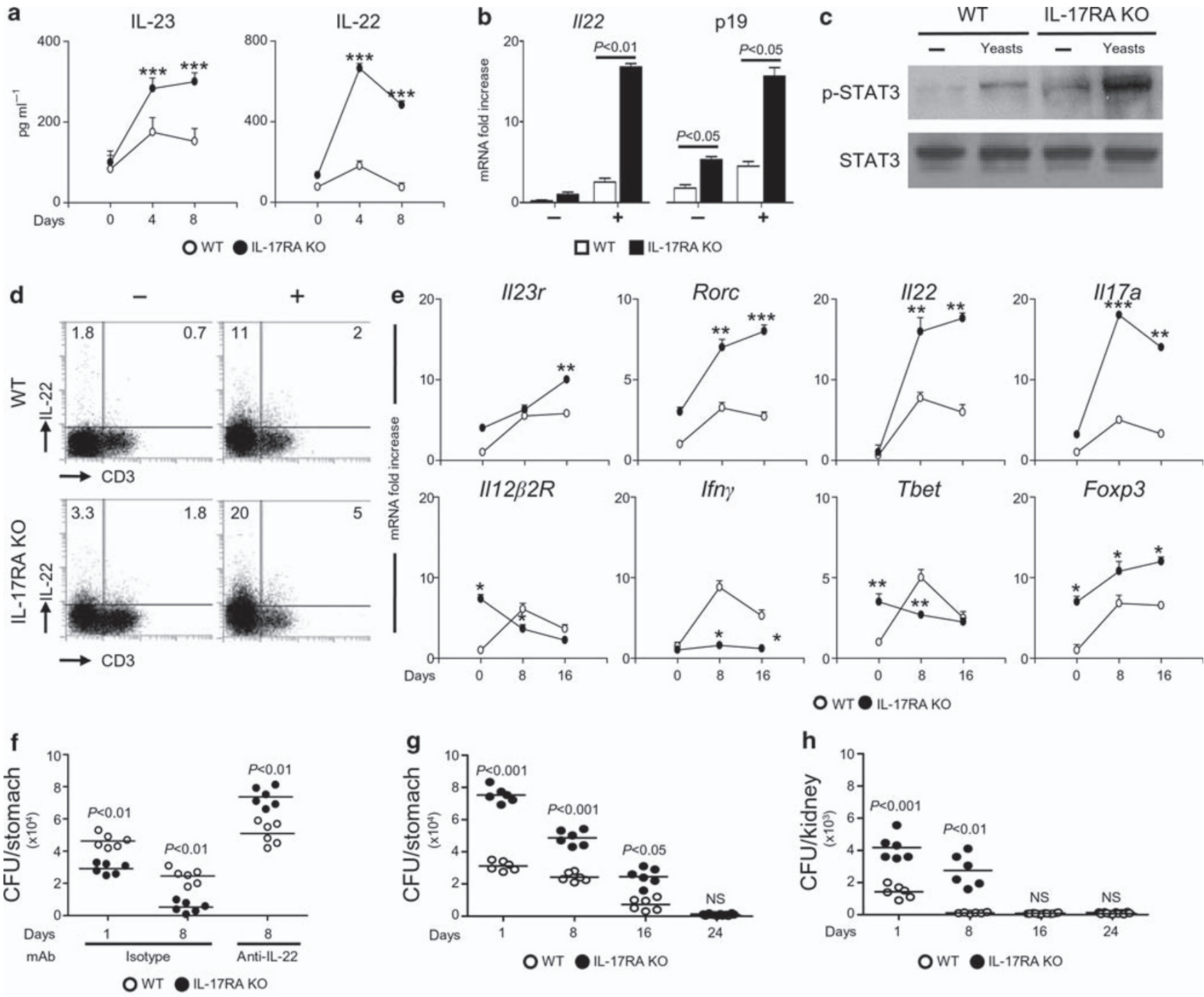

i
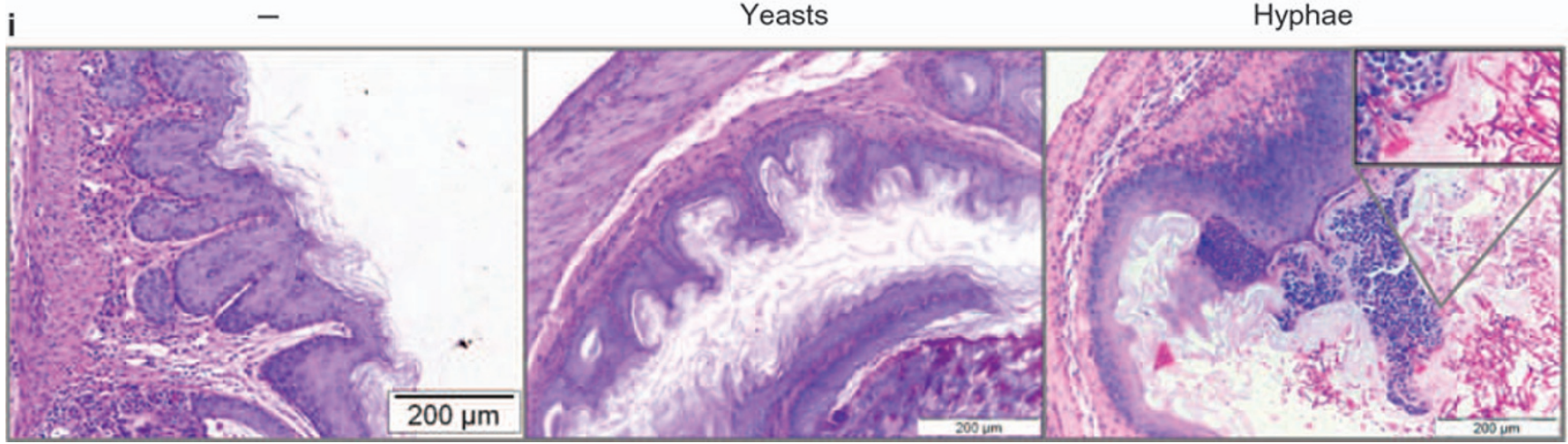

Figure 5 IL-22 is upregulated and Th1 responses are downregulated in IL-17R-deficient mice. (a) IL-23 and IL-22 production (ELISA) in stomach homogenates, (b) II22 and p19 transcripts (real-time RT-PCR) in DCs from Peyers's patches and (c) STAT3 phosphorylation in colonic epithelial cells from C57BL/6 (WT, $n=6$ ) or IL-17RA KO $(n=6)$ mice after intragastric infection with $C$. albicans yeasts. ${ }^{* \star} P<0.001$, KO vs. WT mice. (d) Intracellular staining of IL-22 in CD3-stained mesenteric lymph node a week after infection. Numbers indicate percent cells in gate. (e) Transcripts of II23r, Rorc, II22, II17a, Foxp3, II12 32 r, Tbet and Ifry in CD3 + T cells purified from mesenteric lymph nodes of WT or KO mice infected with $10^{8}$ Candida hyphae. ${ }^{\star} P<0.05,{ }^{\star \star} P<0.01,{ }^{* \star} P<0.001$, KO vs. WT mice. Time-course fungal growth (CFU \pm s.e.) in the stomach of WT ( $\left.n=6\right)$ and IL-17RA KO $(n=6)$ mice inoculated intragastrically with $C$. albicans yeasts (f) or hyphae $(\mathbf{g})$. (h) CFU \pm ES in the kidneys of mice infected as in $\mathrm{G}$. Treatment with IL-22 neutralizing or isotype mAb was done the day of and 1 day after the infection. $P$, KO vs. WT mice and mAb-treated vs. isotype-treated mice. (i) Histological analysis of PAS-stained stomach (7 days after infection) from KO mice uninfected (-) or infected with $C$. albicans yeasts or hyphae. Numerous fungal hyphae and pseudohyphae (magnified in the insets), much longer in KO than WT mice $(41.3 \pm 5.2 \mu \mathrm{m}$ vs. $21.7 \pm 4.5 \mu \mathrm{m})$, were present in the abundant keratinized layer of the stomach of $\mathrm{KO}$ mice. Representative images are shown. Data are representative of four experiments. 

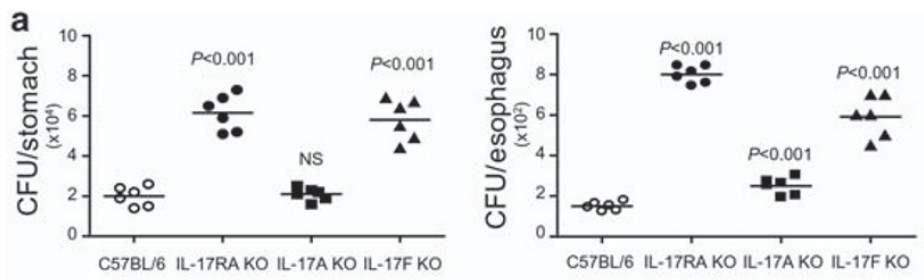

b
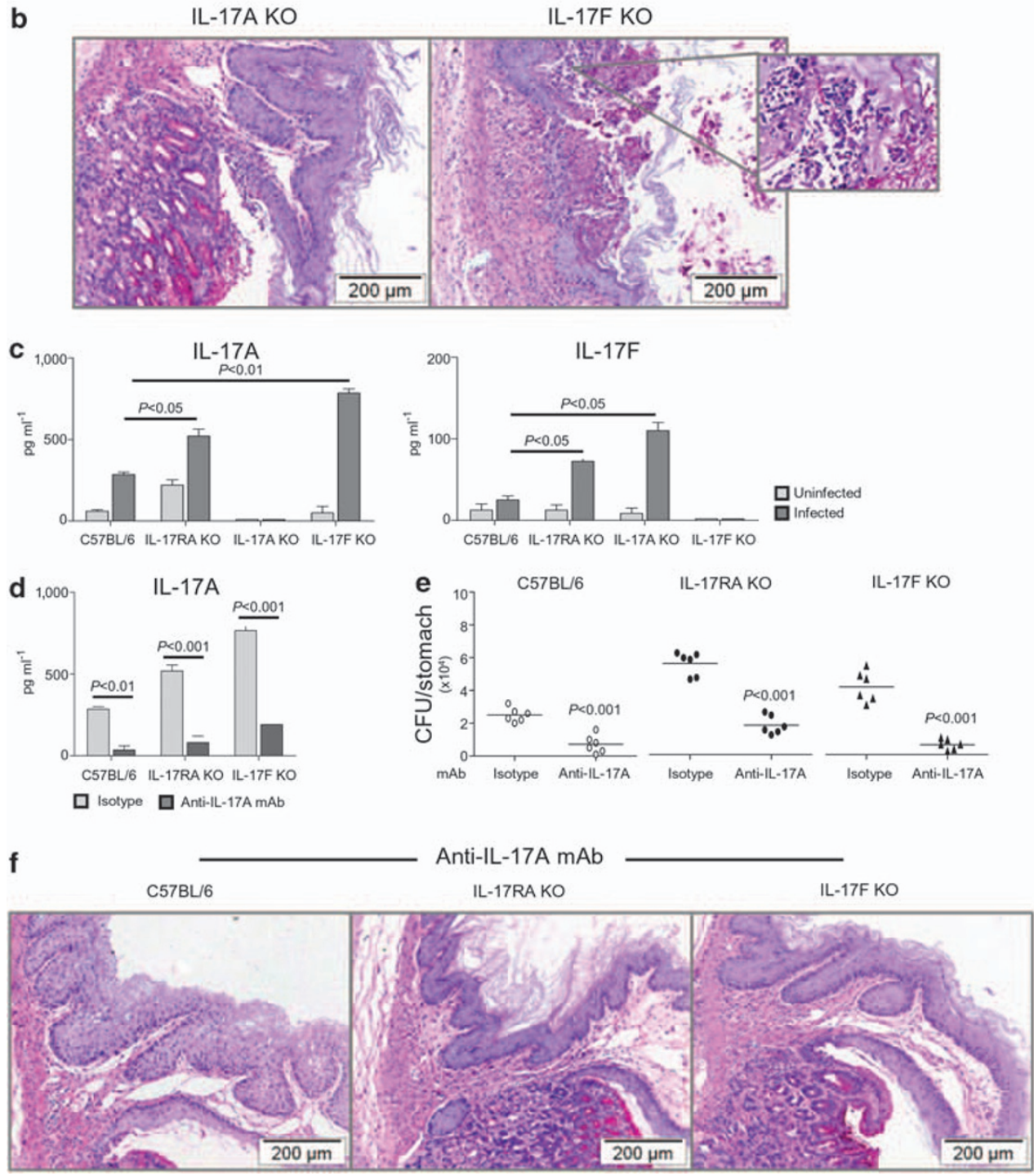

Figure $6 \mathrm{IL}-17 \mathrm{~A}$ and $\mathrm{F}$ are dispensable in gastric candidiasis. (a) Fungal growth (CFU \pm s.e.) in the stomach or esophagus 7 days after the infection of C57BL/6 (WT, $n=6)$, IL-17RA KO $(n=6)$, IL-17A KO $(n=6)$ and IL-17F KO $(n=6)$ mice intragastrically infected with $C$. albicans hyphae. (b) Histological analysis of PAS-stained stomach sections (3 days after the infection) from IL-17A KO or IL-17F KO mice showing reduced inflammatory cell recruitment (magnified in the inset) in IL-17A KO compared with IL-17F KO mice. Representative images are shown. (c) IL-17A and IL-17F productions in uninfected or infected mice and (d) in mice treated with isotype control mAb or IL-17A neutralizing mAb intraperitoneally the day of and 1 day after the infection. $P, \mathrm{KO}$ vs. WT mice and mAb-treated vs. isotype-treated mice. (e) Fungal growth (CFU \pm s.e.) and (f) histology (PAS-stained stomach sections) in mice treated with IL-17A neutralizing mAb intraperitoneally the day of and 1 day after the intragastric infection. Note the amelioration of inflammatory pathology in the stomach upon IL-17A neutralization, as compared with untreated mice (see Figures $\mathbf{3 b} \mathbf{b} \mathbf{5} \mathbf{i}$ and $\mathbf{6} \mathbf{b}$ for the respective untreated controls).

whereas adaptive Th1/Treg cells prevent fungal dissemination and provide memory and tolerance, as already suggested. ${ }^{16}$ Thus, functionally distinct "modules" of immunity evolved to provide resistance, that is, ability to limit fungal burden, or tolerance, that is, the ability to limit local and distal host damage in response to this commensal. ${ }^{3}$ The two pathways, although non-redundant, are reciprocally regulated and compensate each other in the relative absence of either one, consistent with the theme that adaptive immunity depends on innate immunity but innate immunity requires adaptive regulation. ${ }^{37}$ With a functional Th1 pathway, the IL-23/IL-22 axis is even dispensable, an observation that may explain the mild susceptibility to candidiasis 
seen in condition of IL-22 deficiency. ${ }^{8}$ However, in condition of defective Th1 pathway, such as that occurring in IFN- $\gamma$ or IL-17RA deficiency, a heightened innate IL-22 is required for resistance to low infective doses. Ultimately, and consistent with the current view, ${ }^{38-41}$ the commensal nature of the fungus predicts that a defective control of fungal growth rather than the acute exposure to high-dose challenge is associated with human disease.

The reciprocal control between the two pathways may likely occur at the level of IL-23 and IL-12 productions, which are cross-regulated in DCs. ${ }^{2}$ This may account for the decreased early resistance of p19 KO mice to oral, ${ }^{8}$ gastric or systemic candidiasis (this study), a finding confirming the inability of these mice to adequately respond to gut pathogens. ${ }^{42}$ However, Th1-mediated resistance to infection greatly recovered in $\mathrm{p} 19$ KO mice eventually. ${ }^{2}$ In contrast, the failure to activate Th1mediated resistance associated with the failure to control the infection in p35 KO mice, ${ }^{2}$ despite an apparent increased early resistance to oral, ${ }^{8}$ gastric or systemic infection (this study). Thus, the impact of p19 or p35 deficiency largely depends on the stage of the infection.

Despite producing high levels of IL-23/IL-22, p35 KO mice are not as resistant as IFN- $\gamma \mathrm{KO}$ mice to candidiasis and develop inflammatory Th17 responses. ${ }^{2}$ This suggests that a p35dependent mechanism crucially contributes to shape antifungal protective responses downstream of IL-22. This may include the expansion of regulatory Th17 cells, producing more of IL-22 and IL-10 than IL-17A, as seen in IFN- $\gamma$ KO mice. IL-35, made of p35 and Epstein-Barr virus-induced gene 3, the latter known to regulate the expression of IL-17, IL-22 and ROR $\gamma \mathrm{t},{ }^{43}$ is an attractive candidate. Whatever the mechanism, the occurrence of Th17 cells with different effector functions plausibly accommodates within the diversity of Th17 cells. ${ }^{44}$

Of interest, the IL-22 pathway, more than the Th1/Treg pathway, is exploited by nonpathogenic yeasts, such as C. krusei and S. cerevisiae and likely Cryptococcus neoformans, against which a protective effect of IL-23 has been shown. ${ }^{45}$ Considering the burden of nosocomial infections by opportunistically pathogenic yeast species, ${ }^{46}$ our finding may offer new interpretative clues to explain why some individuals are at high risk of yeast infection. That patients with Chronic Mucocutaneous Candidiasis are defective in IL-17 and IL-22 production ${ }^{47}$ is consistent with our findings.

One important finding of this study is that IL-22 mediates protection in IL-17RA KO mice, as indicated by the high resistance of these mice to the infection with Candida yeasts more than hyphae. The high susceptibility to systemic ${ }^{9}$ and oral ${ }^{8}$ candidiasis in condition of defective IL-17RA signaling has been taken to indicate the essential role of IL-17RA signaling in host defense to C. albicans through the ability of IL-17 to mobilize neutrophils and induce $\beta$-defensin-3. However, not only has IL-17A recently been reported to be dispensable in resistance to systemic candidiasis, ${ }^{10}$ but exogenous IL-17 administration failed to rescue the Th17 deficiency and actually caused severe adverse reaction. ${ }^{8}$ We found that IL-17A is dispensable for protection in gastric candidiasis, and actually neutralization of
IL-17A greatly reduced the fungal burden and ameliorated the systemic and the gastrointestinal infections in both IL-17RA KO. IL-17A was elevated in IL-17F KO mice and contributes to susceptibility to the infection in these mice, a finding suggesting that IL-17F by inhibiting IL-17A may exert protective effects in candidiasis, although additional effector activities cannot be ruled out. In addition, the finding that IL-17A blockade increased resistance in IL-17F KO mice clearly indicate that neither cytokine is essential in infection.

We know that the offending potential of the IL-17 family may occur at different levels. ${ }^{2,12}$ First, IL-17A and F greatly promoted the inflammatory activity of neutrophils. ${ }^{2}$ No defective inflammatory cell recruitment was observed in IL-17RA KO mice resisting the infection, a finding suggesting that granulopoiesis may occur independently of IL-17 but that regulation of inflammation is required to cope with the fungus. ${ }^{12}$ Second, a consistent finding of this study was that hyphal growth was greatly promoted at the sites of infection in IL-17RA KO mice. Given the influence of local $\mathrm{pH}$ on morphological transition in C. albicans, we measured the $\mathrm{pH}$ in the stomach of mice with infection and found it lower in IL-17RA KO $(\mathrm{pH}, 2.2)$ than WT $(\mathrm{pH}, 4.4)$ mice. This finding is consistent with the ability of IL-17A to modulate epithelial bicarbonate secretion ${ }^{48}$ and would provide a plausible explanation for the noticeable presence of hyphae in the stomach of IL-17RA KO mice. This observation would also suggest the possible tissue-dependent effect of the IL-17/Th17 axis in infection. Lastly, we have evidence that IL-17A acts on the fungus and promote its fungal growth and virulence (Zelante et al., article in preparation). As the occurrence of protective responses to the fungus are strictly dependent on fungal load, ${ }^{49}$ this may offer a plausible explanation for the suppression of protective responses by IL-17A independently of host IL-17RA. Overall, the offending potential of the IL-17 family, through host-dependent and -independent mechanistic pathways, could account for the increased susceptibility of IL-17RA KO mice to C. albicans hyphae infection, thus masking the ability of these mice to resist infection through IL-22. For C. albicans and certain bacterial infection, ${ }^{14,31}$ a plausible working hypothesis would be that IL-17R signaling in the gut is either organ-dependent or is dispensable for effector host defense and protection against a low microbial load, the control of which is associated with IL-23-dependent IL-22, but not IL-17 production, in the relative absence of Th1 responses. This highlights the IL-23's primary function as first-line defence against environmental assaults, such as in the gut, the lung, and the skin. ${ }^{34}$

In conclusion, our data show the critical role of IL-22 during the early host defense against $C$. albicans and confirm the important immune regulatory role of the Th17 pathway in the induction of optimal Th1 antifungal resistance. ${ }^{3,10,50}$ The new findings may help to explain the susceptibility to bacterial and fungal infections in patients with autosomal-dominant hyper-IgE syndrome (AD-HIES). ${ }^{6,7}$ Owing to dominant-negative mutations of STAT3, ${ }^{51} \mathrm{AD}$-HIES patients have a defective Th17 that is likely amplified on epithelia where STAT3 mutations compromise the IL-22 effects. Thus, the defective Th $1^{52}$ 
in AD-HIES patients and the low IL-22 production in Chronic Mucocutaneous Candidiasis ${ }^{47}$ are clinical features consistent with the Th1-supporting role of and IL-22 production by Th17 cells. However, immune dysfunctions in AD-HIES patients are not solely explained by the loss of Th17 cells. ${ }^{53}$ Finally, the fact that IL-22 production in the gut is driven by commensals also provides a novel mechanistic insight on how antibiotic therapy and iatrogenic immunosuppression are major predisposing factors in candidiasis and, more generally, how bacterial-fungal population dynamics impact gut homeostasis and inflammatory diseases. ${ }^{54}$

\section{METHODS}

\section{Animals}

C57BL/6 and BALB/c mice, 8- to 10-week-old, were purchased from Charles River and Rag2 KO mice from Taconic. Homozygous IL-12p35 $\mathrm{KO}$ or IL-23p19 KO mice on BALB/c or C57BL/6 background, respectively, and IFN- $\gamma$ KO mice on C57BL/6 background, were bred under specific pathogen-free conditions at the Animal Facility of Perugia University, Perugia, Italy. IL-22 KO mice on both BALB/c and C57BL/6 background were as described. ${ }^{27} \mathrm{IL}-17 \mathrm{RA}$ KO mice were from Amgen. Breeder pairs of IL-17A KO and IL-17F KO, generated as described, ${ }^{55}$ were kindly provided by Dr Burkhard Becher, University Hospital of Zurich, $\mathrm{CH}-8057$ Zürich Switzerland. Experiments were performed according to the Italian Approved Animal Welfare Assurance A-3143-01.

\section{Fungal strains and infections}

Isogenic strains of C. albicans, obtained by mutagenesis and capable (referred to as hyphae) or not (referred to as yeasts) of yeast-to-hypha transition, as assessed by the germ-tube formation in vitro, were used. We prepared hyphae by allowing cells to germinate in culture at $37^{\circ} \mathrm{C}$, in $5 \% \mathrm{CO}_{2}$, for $2 \mathrm{~h}$ in RPMI 1640 medium (by that time, $>98 \%$ of cells had germinated) and yeasts, by harvesting the cells at the end of the exponential phase of growth. Candida krusei and Saccharomices cerevisiae were obtained from clinical specimens. We obtained $C$. krusei and Saccharomices cerevisiae from clinical specimens. For gastrointestinal infection, $10^{7}$ or $10^{8}$ C. albicans, $10^{8}$ C. krusei or $S$. cerevisiae were intragastrically injected. Quantification of fungal growth (colony forming units, CFU, per organ, mean \pm s.e.) was done by serially diluting homogenates and plating them in triplicate in Sabouraud agar. For systemic infection, $10^{5}$ or $5 \times 10^{5} \mathrm{C}$. albicans yeasts or hyphae were injected intravenously. For histology, sections $(3-4 \mu \mathrm{m})$ of paraffin-embedded tissues were stained with periodic acid-Schiff (PAS) reagent.

\section{Transmission electron microscopy}

We dissected the stomach to anus for the mice from 4 to $24 \mathrm{~h}$ after the intragastric infection with C. albicans yeasts or hyphae. Thin sections of the gut were fixed in cold $2.5 \%$ glutaraldehyde in $0.1 \mathrm{M}$ of sodium cacodylate $/ 1 \%$ sucrose buffer for $4 \mathrm{~h}$, post-fixed in $1 \%$ osmium tetroxide $(50 \mathrm{~min})$ and dehydrated in a series of graded ethanolic solutions finishing with propylene oxide before finally being embedded in Epon 812-Araldite mixture. Ultrathin sections $(50 \mathrm{~nm})$ were cut on a LKB ultramicrotome and placed under 200 mesh standard copper grids, contrasted with uranyl acetate and lead citrate, and examined with a Philips TEM 400 transmission electron microscope (Eindhoven, The Netherlands).

\section{Blockade of cytokines in vivo}

We intraperitoneally injected a total of 300 (mucosal infection) or 100 (systemic infection) $\mu \mathrm{g}$ of mAb neutralizing IL-23, ${ }^{2}$ IL-17A (R\&D System Clone 50104, rat IgG2a), IL-17F (R\&D System Clone 316016, rat IgG2a), IL-22 (clone AM22.3, mouse IgG2a) and IFN- $\gamma$ (XMG1.2, rat IgG1) the day of and 1 day after the infection. When combined, a total of $100 \mu \mathrm{g}(\mathrm{IL}-17 \mathrm{~A}+\mathrm{IL}-17 \mathrm{~F})$ was injected. Control mice received isotype control $\mathrm{mAb}$.

\section{Cell isolation and culture}

Peyer's patches and colonic epithelial cells were isolated as described, ${ }^{15,35}$ with some modifications. We isolated epithelial cells from colons flushed with HBSS without calcium and magnesium and supplemented with $10 \mu \mathrm{g} \mathrm{ml}^{-1}$ of Fungizone, $100 \mu \mathrm{g} \mathrm{ml}^{-1}$ gentamicin, $100 \mathrm{U} \mathrm{ml}^{-1}$ penicillin, $100 \mu \mathrm{g} \mathrm{ml}^{-1}$ streptomycin and $10 \mathrm{mM} \mathrm{HEPES} \mathrm{(Gibco,} \mathrm{Invitrogen).}$ Colons were cut longitudinally, sectioned into $2-4 \mathrm{~cm}$ pieces, and the pieces were incubated for $90 \mathrm{~min}$ at $37^{\circ} \mathrm{C}$ in RPMI 1640 supplemented as above and containing $2 \% \mathrm{FBS}, 0.25 \mathrm{mg} \mathrm{ml}^{-1}$ Dispase I and $1 \mathrm{mg} \mathrm{ml}^{-1}$ Collagenase type 1 (Sigma) with constant gentle rotation. Epithelial cells were collected after mucus removal and centrifugation and checked for purity by cytokeratin staining. CD11c+ DCs were positively selected from Peyer's patches with MACS microbeads (Miltenyi Biotec, Bergisch Gladbach, Germany). DCs were subsequently exposed to live unopsonized C. albicans for $12 \mathrm{~h}$ before RT-PCR and cytokine quantification in culture supernatants as described. ${ }^{35} \mathrm{CD} 3-\mathrm{NKp} 46+$ were purified from Peyer's patches with FITC-anti-mouse NKp46 Ab (29A1.4 R\&D System) followed by MACS anti-FITC microbeads. We cultured $10^{5} / \mathrm{ml} \mathrm{NKp} 46+$ cells in anti-NKp46 $\left(5 \mu \mathrm{g} \mathrm{ml}^{-1}\right)$-coated U-bottomed 96-well plates (Immulon) in RPMI-1640 medium containing 2\% heat-inactivated FBS (Hyclone), $2.5 \mathrm{mg} \mathrm{ml}^{-1}$ of Fungizone, $10 \mathrm{mg} \mathrm{ml}^{-1}$ Gentamicin, $100 \mathrm{U} \mathrm{ml}^{-1}$ Penicillin, $100 \mu \mathrm{g} \mathrm{ml}^{-1}$ Streptomycin and $10 \mathrm{~mm}$ HEPES (Gibco, Invitrogen) with live unopsonized C. albicans (1:1 ratio) in the presence of $20 \mathrm{ng}$ rIL-23 (R\&D System) ${ }^{17}$ for $18 \mathrm{~h}$ before IL-22 determination in the supernatants. $\mathrm{CD} 4+, \mathrm{CD} 8+$ or $\mathrm{CD} 3+$ cells were purified from mesenteric lymph nodes with MACS microbeads and restimulated with plate bound anti-CD $3 \varepsilon\left(2 \mu \mathrm{g} \mathrm{ml}^{-1}\right)$ and anti-CD28

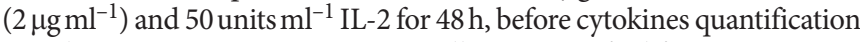
in culture supernatants. IL-17A + cells were purified from mesenteric lymph nodes, after in vitro re-stimulation as per the manufacturer's instruction, with the mouse IL-17 Secretion Assay (Miltenyi Biotec).

\section{Intracellular staining}

For intracellular cytokine detection, $10^{7}$ total mesenteric lymph node cells or purified CD4 + and CD8 + cells were stimulated with $200 \mathrm{ng} \mathrm{ml}^{-1}$ of phorbol 12-myristate 13 -acetate (Sigma) and $1 \mu \mathrm{g} \mathrm{ml}^{-1}$ of ionomycin (Sigma) for $2 \mathrm{~h}$ at $37^{\circ} \mathrm{C}$. Cells were cultured for four additional hours with $1 \mu \mathrm{g} \mathrm{ml}^{-1}$ brefeldin and then fixed and permeabilized with the Cytofix/Cytoperm Plus Fixation/permeabilization Kit (BD PharMingen). Cells were stained with fluorochrome-labelled antibodies anti-IL-22 (clone 14.03.01, R\&D System) or anti-IL-17A (clone: eBio 17B7, eBioscience) and analyzed on a FACSCan using the Cell Quest program (BD PharMingen).

\section{Western blotting}

Epithelial cells were seeded at $10^{6} / \mathrm{ml}$ in RPMI-1640 medium supplemented as above and stimulated with live unopsonized $C$. albicans yeasts or hyphae (1:1 ratio) in the presence of $20 \mathrm{ng}$ rIL-22 (R\&D System) for $15 \mathrm{~min}$ at $37^{\circ} \mathrm{C}$. STAT3 phosphorylation was assessed as described ${ }^{35}$ (see Supplementary Experimental Procedures).

\section{ELISA assay}

Cytokine content was determined by enzyme-linked immunosorbent assays (BD Biosciences Pharmingen, R\&D Systems and eBioscience) on tissue homogenates or supernatants of cultured cells. The detection limits $\left(\mathrm{pg} \mathrm{ml}^{-1}\right)$ of the assays were $<3.2$ for IL-22, $<10$ for IL-12p70, $<30$ for IL-23, $<10$ for IFN- $\gamma,<10$ for IL- $4,<3$ for IL-10, $<10$ for IL-17A and IL-17F.

\section{RNA isolation and reverse transcriptase PCR}

Cell and tissue RNA was obtained with the RNeasy Mini Kit (Qiagen S.p.A., Milano, Italy). Il10 primers and RT-PCR were as described. ${ }^{16}$ We performed real-time RT-PCR using the iCycler iQ detection system (Bio-Rad), SYBR Green chemistry (Finnzymes Oy, Espoo, Finland) and Sensiscript reverse transcriptase (Qiagen). PCR primers (Supplementary Experimental Procedures) were from Invitrogen. 
Samples were normalized to the housekeeping Gapdh gene and were expressed as fold increase over day 0 or mock-infected cells.

\section{Statistical analysis}

We calculated statistical significance by one- or two-way ANOVA or paired $t$-test with Prism software (GraphPad). We considered all $P$-values $\leqslant 0.05$ significant. Data are representative of at least two independent experiments or pooled from three to five experiments. The in vivo groups consisted of six mice/group.

\section{DISCLOSURE}

The authors declared no conflict of interest.

SUPPLEMENTARY MATERIAL is linked to the online version of the paper at http://www.nature.com/mi

\section{ACKNOWLEDGMENTS}

We thank Dr Cristina Massi Benedetti for editorial assistance. This study was supported by the Specific Targeted Research Project SYBARIS (FP7-Health2009-single-stage, contract number 242220) and the Italian Projects PRIN 2007KLCKP8_004 (to LR) and PRIN 2007KLCKP8_005 (to FB).

(c) 2010 Society for Mucosal Immunology

\section{REFERENCES}

1. Calderone, R.A. Candida and Candidiasis (ASM Press, Washington, DC, USA, 2002).

2. Zelante, T. et al. IL-23 and the Th17 pathway promote inflammation and impair antifungal immune resistance. Eur. J. Immunol. 37, 2695-2706 (2007).

3. Zelante, T., De Luca, A., D'Angelo, C., Moretti, S. \& Romani, L. IL-17/ Th17 in anti-fungal immunity: what's new? Eur. J. Immunol. 39, 645-648 (2009).

4. Acosta-Rodriguez, E.V. et al. Surface phenotype and antigenic specificity of human interleukin 17-producing T helper memory cells. Nat. Immunol. 8, 639-646 (2007).

5. de Beaucoudrey, L. et al. Mutations in STAT3 and IL12RB1 impair the development of human IL-17-producing T cells. J. Exp. Med. 205, 1543-1550 (2008).

6. Ma, C.S. et al. Deficiency of Th17 cells in hyper lgE syndrome due to mutations in STAT3. J. Exp. Med. 205, 1551-1557 (2008).

7. Milner, J.D. et al. Impaired $\mathrm{T}(\mathrm{H}) 17$ cell differentiation in subjects with autosomal dominant hyper-IgE syndrome. Nature 452, 773-776 (2008).

8. Conti, H.R. et al. Th17 cells and IL-17 receptor signaling are essential for mucosal host defense against oral candidiasis. J. Exp. Med. 206, 299-311 (2009).

9. Huang, W., Na, L., Fidel, P.L. \& Schwarzenberger, P. Requirement of interleukin-17A for systemic anti-Candida albicans host defense in mice. J. Infect. Dis. 190, 624-631 (2004).

10. Lin, L. et al. Th1-Th17 cells mediate protective adaptive immunity against Staphylococcus aureus and Candida albicans infection in mice. PLoS Pathog. 5, e1000703 (2009).

11. Bozza, S. et al. Lack of toll IL-1R8 exacerbates Th17 cell responses in fungal infection. J. Immunol. 180, 4022-4031 (2008).

12. Romani, L., Zelante, T., De Luca, A., Fallarino, F. \& Puccetti, P. IL-17 and therapeutic kynurenines in pathogenic inflammation to fungi. J. Immunol. 180, 5157-5162 (2008).

13. Aujla, S.J. et al. IL-22 mediates mucosal host defense against Gramnegative bacterial pneumonia. Nat. Med. 14, 275-281 (2008).

14. Schulz, S.M. et al. Protective immunity to systemic infection with attenuated Salmonella enterica serovar enteritidis in the absence of IL-12 is associated with IL-23-dependent IL-22, but not IL-17. J. Immunol. 181, 7891-7901 (2008)

15. Zheng, Y. et al. Interleukin-22 mediates early host defense against attaching and effacing bacterial pathogens. Nat. Med. 14, 282-289 (2008).

16. De Luca, A. et al. Functional yet balanced reactivity to Candida albicans requires TRIF, MyD88, and IDO-dependent inhibition of Rorc. J. Immunol. 179, 5999-6008 (2007).
17. Cella, M. et al. A human natural killer cell subset provides an innate source of IL-22 for mucosal immunity. Nature 457, 722-725 (2009).

18. Luci, C. et al. Influence of the transcription factor RORgammat on the development of NKp46+ cell populations in gut and skin. Nat. Immunol. 10, 75-82 (2009).

19. Sanos, S.L. et al. RORgammat and commensal microflora are required for the differentiation of mucosal interleukin 22-producing NKp46+ cells. Nat. Immunol. 10, 83-91 (2009).

20. Satoh-Takayama, N. et al. Microbial flora drives interleukin 22 production in intestinal NKp46+ cells that provide innate mucosal immune defense. Immunity 29, 958-970 (2008).

21. Vivier, E., Spits, H. \& Cupedo, T. Interleukin-22-producing innate immune cells: new players in mucosal immunity and tissue repair? Nat. Rev. Immunol. 9, 229-234 (2009).

22. Veldhoen, M. et al. The aryl hydrocarbon receptor links TH17-cell-mediated autoimmunity to environmental toxins. Nature 453, 106-109 (2008).

23. Takatori, H. et al. Lymphoid tissue inducer-like cells are an innate source of IL-17 and IL-22. J. Exp. Med. 206, 35-41 (2009).

24. Liang, S.C. et al. Interleukin (IL)-22 and IL-17 are coexpressed by Th17 cells and cooperatively enhance expression of antimicrobial peptides. J. Exp. Med. 203, 2271-2279 (2006).

25. Kolls, J.K., McCray, P.B. Jr. \& Chan, Y.R. Cytokine-mediated regulation of antimicrobial proteins. Nat. Rev. Immunol. 8, 829-835 (2008).

26. Wolk, K. et al. IL-22 increases the innate immunity of tissues. Immunity $\mathbf{2 1}$, 241-254 (2004).

27. Geboes, L. et al. Proinflammatory role of the Th17 cytokine interleukin-22 in collagen-induced arthritis in C57BL/6 mice. Arthritis Rheum. 60, 390-395 (2009).

28. Lochner, M. et al. In vivo equilibrium of proinflammatory IL-17+ and regulatory IL-10+ Foxp3+ RORgamma t+ T cells. J. Exp. Med. 205, 1381-1393 (2008).

29. Zhou, L. et al. TGF-beta-induced Foxp3 inhibits $T(H) 17$ cell differentiation by antagonizing RORgammat function. Nature 453, 236-240 (2008).

30. Ma, H.L. et al. IL-22 is required for Th17 cell-mediated pathology in a mouse model of psoriasis-like skin inflammation. J. Clin. Invest. 118, 597-607 (2008).

31. Khader, S.A. \& Cooper, A.M. IL-23 and IL-17 in tuberculosis. Cytokine 41, 79-83 (2008).

32. Lee, Y.K. et al. Late developmental plasticity in the T helper 17 lineage. Immunity 30, 92-107 (2009).

33. Nagata, T. et al. Requirement of IL-17RA in Con A induced hepatitis and negative regulation of IL-17 production in mouse T cells. J. Immunol. 181, 7473-7479 (2008)

34. Tato, C.M. \& Cua, D.J. Reconciling id, ego, and superego within interleukin-23. Immunol. Rev. 226, 103-111 (2008).

35. Bonifazi, P. et al. Balancing inflammation and tolerance in vivo via dendritic cells by the commensal Candida albicans. Mucosal Immunol 2, 362-374 (2009).

36. Xu, T., Logsdon, N.J. \& Walter, M.R. Structure of insect-cell-derived IL-22. Acta Crystallogr D Biol. Crystallogr. 61, 942-950 (2005).

37. Kim, K.D. et al. Adaptive immune cells temper initial innate responses. Nat. Med. 13, 1248-1252 (2007).

38. Casadevall, A. \& Pirofski, L.A. Accidental virulence, cryptic pathogenesis, martians, lost hosts, and the pathogenicity of environmental microbes. Eukaryot. Cell 6, 2169-2174 (2007).

39. Pirofski, L.A. \& Casadevall, A. Rethinking T cell immunity in oropharyngeal candidiasis. J. Exp. Med. 206, 269-273 (2009).

40. Romani, L., Bistoni, F. \& Puccetti, P. Adaptation of Candida albicans to the host environment: the role of morphogenesis in virulence and survival in mammalian hosts. Curr. Opin. Microbiol. 6, 338-343 (2003).

41. Romani, L. \& Puccetti, P. Protective tolerance to fungi: the role of IL-10 and tryptophan catabolism. Trends Microbiol. 14, 183-189 (2006).

42. Mangan, P.R. et al. Transforming growth factor-beta induces development of the $T(H) 17$ lineage. Nature 441, 231-234 (2006).

43. Yang, J. et al. Epstein-Barr virus-induced gene 3 negatively regulates IL-17, IL-22 and RORgamma t. Eur. J. Immunol. 38, 1204-1214 (2008).

44. Spolski, R. \& Leonard, W.J. Cytokine mediators of Th17 function. Eur. J. Immunol. 39, 658-661 (2009).

45. Kleinschek, M.A. et al. IL-23 enhances the inflammatory cell response in Cryptococcus neoformans infection and induces a cytokine pattern distinct from IL-12. J. Immunol. 176, 1098-1106 (2006). 
46. Peres-Bota, D., Rodriguez-Villalobos, H., Dimopoulos, G., Melot, C. \& Vincent, J.L. Potential risk factors for infection with Candida spp. in critically ill patients. Clin. Microbiol. Infect. 10, 550-555 (2004).

47. Eyerich, K. et al. Patients with chronic mucocutaneous candidiasis exhibit reduced production of Th17-associated cytokines IL-17 and IL-22. J. Invest Dermatol. 128, 2640-2645 (2008).

48. Kreindler, J.L. et al. Interleukin-17A induces bicarbonate secretion in normal human bronchial epithelial cells. Am. J. Physiol. Lung Cell Mol. Physiol. 296, L257-L266 (2009).

49. Mencacci, A. et al. CD4+ T-helper-cell responses in mice with low-level Candida albicans infection. Infect Immun. 64, 4907-4914 (1996).

50. Cooper, A.M. IL-23 and IL-17 have a multi-faceted largely negative role in fungal infection. Eur. J. Immunol. 37, 2680-2682 (2007).
51. Holland, S.M. et al. STAT3 mutations in the hyper-lgE syndrome. N Engl. J. Med. 357, 1608-1619 (2007).

52. Netea, M.G., Kulliberg, B.J. \& van der Meer, J.W. Severely impaired IL-12/ IL-18/IFNgamma axis in patients with hyper lgE syndrome. Eur. J. Clin. Invest. 35, 718-721 (2005).

53. Tangye, S.G., Cook, M.C. \& Fulcher, D.A. Insights into the role of STAT3 in human lymphocyte differentiation as revealed by the hyper-IgE syndrome. J. Immunol. 182, 21-28 (2009).

54. Wargo, M.J. \& Hogan, D.A. Fungal-bacterial interactions: a mixed bag of mingling microbes. Curr. Opin. Microbiol. 9, 359-364 (2006).

55. Haak, S. et al. IL-17A and IL-17F do not contribute vitally to autoimmune neuro-inflammation in mice. J. Clin. Invest. 119, 61-69 (2009). 